\section{CORRELATION OF EEG-BASED BRAIN RESUSCITATION INDEX(EBRI) AND END TIDAL CO2 IN PORCINE CARDIAC ARREST MODEL: EBRI-ONE TRIAL}

${ }^{1}$ Dong Sun Choi, ${ }^{2}$ Heejin Kim, ${ }^{1}$ So-Ra Song, ${ }^{3}$ Tae Han Kim, ${ }^{1}$ Young Sun Ro, ${ }^{3} \mathrm{Ki}$ Jeong Hong, ${ }^{4}$ Kyungjun Song, ${ }^{4}$ Jung ho Park, ${ }^{2}$ Hee Chan Kim, ${ }^{4}$ Sang Do Shin*. ${ }^{1}$ Laboratory of Emergency Medical Services, Seoul National University Hospital Biomedical Research Institute, Republic of Korea; ${ }^{2}$ Institute of Medical and Biological Engineering, Medical Research Centre, Department of Biomedical Engineering, Seoul National University College of Medicine, Seoul, Republic of Korea; ${ }^{3}$ Department of Emergency Medicine, Seoul National University Boramae Medical Centre, Seoul, Republic of Korea; ${ }^{4}$ Department of Emergency Medicine, Seoul National University Hospital, Seoul, Republic of Korea

\subsection{6/10.1136/bmjopen-2018-EMS.47}

Aim Evaluation and monitoring of brain viability is important during resuscitation of cardiac arrest. We developed non-invasive EEG-based brain resuscitation index (EBRI) and evaluated correlation EBRI and end-tidal CO2(ETCO2).

Method A randomised crossover animal experimental study using porcine cardiac arrest model was designed. After 1 min of untreated ventricular fibrillation, alternation of high quality CPR (compression depth $5 \mathrm{~cm}$ and compression rate 100/min) and low quality CPR (compression depth $3 \mathrm{~cm}$ and compression rate $60 / \mathrm{min}$ ) was performed for every $50 \mathrm{~s}$ in 10 phases. EBRI was calculated from selected single EEG channel which have the lowest noise. Mixed model analysis was conducted to compare the differences of haemodynamic parameters, ETCO2 and EBRI between high quality CPR period and low quality CPR period. Pearson's correlation coefficient was calculated to assess correlation between EBRI and ETCO2.

Results Experiment was performed in four female porcine $(44.6 \pm 2.8 \mathrm{~kg})$. EBRI and EtCO2 was obtained according to quality of CPR received. Delta ETCO2-based EBRI obtained during high quality CPR was significantly higher than delta EBRI of lower quality CPR (HQ : Median 0.1, (0.0-0.2), LQ: Median $-0.1(-0.2-0.0, \mathrm{p} \leq 0.01)$. EBRI had statistically moderate positive correlation with ETCO2 $(r=0.51)$.

Conclusion In porcine cardiac arrest model, EEG-based Brain Resuscitation Index was successfully obtained during resuscitation and had statistically moderate correlation with ETCO2.

Conflict of interest None

Funding None

\section{TRENDS IN THE INCIDENCE AND OUTCOME OF PAEDIATRIC OUT-OF-HOSPITAL CARDIAC ARREST IN VICTORIA, AUSTRALIA}

1,2Z Nehme, ${ }^{3} \mathrm{~S}$ Namachivayam, ${ }^{3} \mathrm{~W}$ Butt, ${ }^{1,2,4} \mathrm{~S}$ Bernard, ${ }^{1,2,5} \mathrm{~K}$ Smith*. ${ }^{2}$ Ambulance Victoria, Australia; ${ }^{2}$ Monash University, Australia; ${ }^{3}$ Royal Children's Hospital Melbourne, Australia; ${ }^{4}$ Alfred Hospital, Australia; ${ }^{5}$ University of Western Australia, Australia

\subsection{6/10.1136/bmjopen-2018-EMS.48}

Aim System-based improvements to the chain of survival have yielded significant increases in survival from out-of-hospital cardiac arrest (OHCA) in adults. Comparatively little is known about the long-term trends in incidence and survival following paediatric OHCA.

Method Between 2000 and 2016, we included paediatrics aged $\leq 16$ years who suffered a non-traumatic OHCA in the state of Victoria, Australia. Trends in incidence and unadjusted outcomes were assessed using linear regression. Adjusted trends in event survival and survival to hospital discharge were assessed using multivariable logistic regression.
Results Of the 1301 paediatric OHCA attended by emergency medical services (EMS), 948 (72.9\%) received an attempted resuscitation. The overall incidence of EMS-attended OHCA was 6.7 cases per 100000 person-years, with no significant change over time. Although median EMS response times increased over time, the proportion of cases with OHCA identified in the call and receiving bystander cardiopulmonary resuscitation (CPR) also increased. Unadjusted event survival rose from $23.3 \%$ in 2000 to $33.3 \%$ in 2016 ( $\mathrm{p}$ trend $=0.007$ ). Over the same period, survival to hospital discharge rose from $9.4 \%$ to $17.7 \%$ ( $\mathrm{p}$ trend $=0.04$ ). After multivariable adjustment, the odds of event survival and survival to hospital discharge increased independently of arrest factors, by 7\% (OR 1.07, 95\% CI: 1.03, 1.12; p=0.001) and $8 \%$ (OR 1.08, 95\% CI: 1.01, 1.15; p=0.02) respectively. Bystander CPR and OHCA identification in the call were not associated with survival.

Conclusion In our region, survival following paediatric OHCA increased significantly over a 17 year period. However, the factors contributing to this improvement require further investigation.

Conflict of interest None

Funding $\mathrm{ZN}$ is funded by a National Health and Medical Research Council (NHMRC) Early Career Fellowship (\#1146809).

\section{EFFECT OF HYPOTHERMIA ON OUT-OF-HOSPITAL CARDIAC ARREST PATIENT WITH CHRONIC KIDNEY DISEASE}

${ }^{1}$ Min Woo Kim*, ${ }^{2}$ Young Sun Ro, ${ }^{1}$ Sang Do Shin, ${ }^{1}$ Kyoung Jun Song, ${ }^{1}$ Ki Jeong Hong, ${ }^{1}$ Jung Ho Park. 'Department of Emergency Medicine, Seoul National University College of Medicine, South Korea; 'Laboratory of Emergency Medical Services, Seoul National University Hospital, South Korea

\subsection{6/10.1136/bmjopen-2018-EMS.49}

Aim Therapeutic hypothermia is performed to improve the OHCA patient's neurologic outcome. It is unclear whether the effect size of therapeutic hypothermia differs according to the baseline kidney function.

Method OHCA patients transported to ED from 2013 to 2015 in South Korea were analysed. OHCA Patients due to cardiac cause at age 15 or older, and who survived to admission were included. Those who did not get CPR or without information about neurologic outcome (CPC) at discharge were excluded. Main Exposure was whether therapeutic hypothermia was done at the hospital. Primary and secondary outcomes were hospital survival and CPC score. Multivariable logistic regression was used to calculate the adjusted odds ratios with $95 \%$ confidence intervals. To compare the effect size of the therapeutic hypothermia by underlying kidney dysfunction (CKD-nonHD, HD) and normal kidney function, interaction term (hypothermia*CKD) was added to the final model.

Results Totally 8170 patients were analysed. 4655 (56.98\%) patients died and 3515 (43.02\%) survived to discharge. Only 2118 (25.92\%) had good neurologic outcome. The AOR of hypothermia were $1.70(1.50-1.9)$ for the survival rate and $1.00(0.88-1.18)$ for the favourable neurologic outcome. In interaction model, the AORs for survival rate of hypothermia were 1.76 (1.54-2.02), $1.27(0.59-2.74)$ and $1.8(0.60-1.96)$ in non-CKD, CKD-nonHD, and HD group respectively. Also, the AORs for good neurologic outcome of hypothermia were $1.08(0.93-1.25), 0.37(0.10-1.42)$ and $0.26(0.09-0.73)$, respectively. 
Conclusion Therapeutic Hypothermia on OHCA patients with underlying kidney dysfunction was less effective than with normal kidney function.

Conflict of interest None

Funding The study was financially supported by the Korea Centres for Disease Control and Prevention (CDC).

\section{CHARACTERISTICS OF PAEDIATRIC TRAUMA PATIENTS ATTENDED BY EMERGENCY MEDICAL SERVICES IN VICTORIA, AUSTRALIA}

${ }^{1}$ Renee Roggenkamp, 1,2Shelley Cox, 1,2Emily Andrew, 1,2,3 Stephen Bernard, 1,2,4Karen Smith*. 'Centre for Research and Evaluation, Ambulance Victoria, Victoria; ${ }^{2}$ Department of Epidemiology and Preventive Medicine, Monash University, Victoria; ${ }^{3}$ Intensive Care Unit, The Alfred Hospital, Victoria; ${ }^{4}$ Department of Community Emergency Health and Paramedic Practice, Monash University, Victoria

10.1136/10.1136/bmjopen-2018-EMS.50

Aim Few studies have described the epidemiological profile of paediatric trauma patients attended by emergency medical services (EMS). This study describes the characteristics of paediatric trauma patients attended by EMS in Victoria, Australia.

Method A retrospective review was conducted of all paediatric trauma patients ( $\leq 15$ years) attended by EMS in Victoria, Australia between 1 July 2012 and 30 June 2017. Descriptive analyses were conducted.

Results Paediatric trauma patients represented 2.2\% of the EMS emergency caseload during the study period. Most patients were male $(59.9 \%)$, and the median age was 8 years. The most common final paramedic diagnoses were pain (21.4\%), lacerations $(14.1 \%)$ and fractures $(12.5 \%)$. Falls, sporting injuries and traffic-related trauma were the most common mechanisms of injury. The majority of patients were transported to hospital (69.5\%) and 55.9\% received clinical treatment by paramedics. A total of $19.3 \%$ of patients were transported to the paediatric major trauma facility in Victoria. Analgesia was administered to $57.9 \%$ of treated patients, with administration increasing with age. A splint was applied most commonly to patients aged 5 to 12 years, and spinal immobilisation was most commonly used in patients aged 13 to 15 years. Patients aged $\leq 4$ years had the highest proportion of patients with an initial pain score of 0 (49.5\%), indicating no pain.

Conclusion The characteristics of paediatric trauma patients differ according to age. Pain, lacerations and fractures are the most common reasons for EMS attendance. Understanding the characteristics of paediatric trauma patients may assist in refining protocols to improve patient outcomes.

Conflict of interest None

Funding None

\section{EVALUATION OF AMBULANCE DISPATCH WHEN TWO TRIAGE SCALES ARE USED IN THE PRE-HOSPITAL SYSTEM}

\footnotetext{
1,2Veronica Lindström, ${ }^{3}$ Blake Langlais, ${ }^{4,5} \mathrm{Glenn}$ Larsson, ${ }^{6}$ Lizbet Todorova,

2,7 Katarina Bohm*. 'Karolinska Institutet, Department of neurobiology, care sciences and society, Division of nursing and Academic EMS in Stockholm, Sweden; ${ }^{2}$ Karolinska Institutet, Department of clinical science and education, Södersjukhuset; ${ }^{3}$ Phoenix, Arizona, USA; ${ }^{4}$ Department of Ambulance and Prehospital Care, Region Halland, Sweden; ${ }^{5}$ Department of Orthopaedics, Lund University, Sweden; ${ }^{6}$ Region Skåne Prehospital Unit, Region Skåne, Sweden; ${ }^{7}$ Department of Emergency medicine, Södersjukhuset, Stockholm
}

Aim Most of the Emergency medical communication centres (EMCC) in Sweden uses the criteria based dispatch protocol (CBD), Medical Index ${ }^{1}$ which has three levels of priority. In the ambulance service, RETTS (Rapid Emergency Triage and Treatment System) $)^{2}$ is used and consists of four levels. This study evaluates the accuracy of dispatching ambulances, by evaluating the feedback sent from the ambulance crew after the first evaluation of the patients' severity and condition.

Method This is a retrospective study from October 2014 to June 2015. Sensitivity and specificity were used to estimate the agreement of assessed priority levels between ambulance priority Red to EMCC priority 1 . The priority level assessed by ambulance crew was considered the 'gold standard.' Over and under triage was described for the six most frequent conditions assessed.

Results There were 66983 missions during the study period. Sensitivity for detecting highest priority indicated $67.1 \%$ correctly identified (95\% confidence interval (CI): 65.6 to 68.7) and specificity indicated $61.8 \%$ of non-highest priority missions not dispatched as priority 1 (95\% CI: 61.2 to 62). Consistency of condition between EMCC and ambulance for the six most common dispatched assignments was: chest pain $82 \%$, minor injuries $81 \%$, stroke $78 \%$, breathing difficulties $74 \%$, abdomen $71 \%$, and undefined problems $29 \%$. The rate of over- and under triage for highest priority was $33.49 \%$ and $4.02 \%$ respectively.

Conclusion Compared to the first evaluation of the patients' severity and condition by the ambulance crew, EMCC assessment had moderate sensitivity and specificity. The lack of universal metrics is a limitation when concluding dispatch accuracy.

\section{REFERENCES}

1. Andersen MS, Johnsen SP, Sørensen JN, Jepsen SB, Hansen JB, Christensen EF Implementing a nationwide criteria-based emergency medical dispatch system: A register-based follow-up study. Scand I Trauma Resusc Emerg Med 2013;21:53.

2. Predicare. Rapid Emergency Triage and Treatment System (REETS): Predicare $A B$. [Available from: http://predicare.se/en/].

Conflict of interest None

Funding None

\section{REGISTRATION OF PREHOSPITAL VITAL PARAMETERS}

${ }^{1} \mathrm{NR}$ Poulsen*, ${ }^{1} \mathrm{TA}$ Kløjgård, ${ }^{1} \mathrm{H}$ Holdgaard, ${ }^{1} \mathrm{E}$ Færk, ${ }^{1,2} \mathrm{TM}$ Larsen, ${ }^{1,3,4} \mathrm{EF}$ Christensen. ${ }^{1}$ Department of Clinical Medicine, Centre for Prehospital and Emergency Research, Aalborg University, Aalborg, Denmark; ${ }^{2}$ Unit of Business Intelligence, North Denmark Region, Aalborg, Denmark; ${ }^{3}$ Department of Anaesthesiology and Intensive Care, Aalborg University Hospital, Aalborg, Denmark; ${ }^{4}$ Emergency Medical Services, North Denmark Region, Aalborg, Denmark

\subsection{6/10.1136/bmjopen-2018-EMS.52}

Aim The North Denmark Region implemented an electronic Prehospital Patient medical Record (PPR) in 2006. In 2015 a new version of PPR was implemented. Implementation of new technologies can be challenging, including structurally and organisational obstacles, ${ }^{1}$ which causes difficulty in achieving data completeness. We aimed to examine registrations of vital parameters in PPR before and after the new version of PPR.

Method The cohort includes all patients to whom an ambulance was dispatched after an emergency 112-call in the North Denmark Region from 2007-2014 and 2016. We examined the distribution and registration of the first measurement of vital parameters.

Results We identified 213.466 patients. Percentage of registrations from 2007 to 2014 vs 2016: Blood pressure (BP) $73 \%$ 